\title{
Dor pediátrica: percepções da equipe médica
}

\author{
Pediatric pain: perceptions of the medical team \\ Dolor pediátrico: percepciones del equipo médico
}

Rená Alves ${ }^{1 *}$, Samara Bertin Suguitani Santello ${ }^{1}$ Andressa Ferreira Adão

\section{RESUMO}

Objetivo: Identificar a percepção de médicos acerca do manejo da dor e os critérios para a escolha analgésica em pacientes pediátricos e neonatais hospitalizados em unidades de terapia intensiva pediátrica e neonatal. Métodos: Estudo descritivo, qualitativo, realizado no ano de 2020 em uma Unidade de Terapia Intensiva Pediátrica (UTIP) e uma Unidade de Terapia Intensiva Neonatal (UTINEO) de um Hospital Regional de nível terciário vinculado a uma universidade privada do interior do estado de São Paulo. Para a realização das entrevistas, foi utilizada uma entrevista semiestruturada e analisada segundo Análise de Conteúdo proposta por Bardin. Resultados: Os dados foram categorizados em duas categorias temáticas, sendo elas: "Como percebo a dor?" e "A analgesia e o manejo da dor". Nas falas foram contemplados pontos como a percepção da dor pediátrica, os instrumentos que utilizam para avaliação da dor e as estratégias para 0 alívio. Conclusão: Possibilitou identificar que os pediatras e neonatologistas estão sensibilizados com o manejo da dor pediátrica, se preocupam e prescrevem analgesia considerando o escalonamento das medicações, 0 quadro clínico e as particularidades de cada indivíduo. Porém, foi possível identificar que alguns participantes não utilizam instrumentos de avaliação da dor pediátrica.

Palavras-chave: Dor, Analgesia, Pediatria.

\begin{abstract}
Objective: To identify the perception of doctors about pain management and the criteria for analgesic choice in pediatric and neonatal patients hospitalized in pediatric and neonatal intensive care units. Methods: Descriptive, qualitative study, carried out in 2020 in a Pediatric Intensive Care Unit (PICU) and a Neonatal Intensive Care Unit (UTINEO) of a tertiary-level Regional Hospital linked to a private university in the state of São Paulo Paulo. To conduct the interviews, a semi-structured interview was used and analyzed according to Content Analysis proposed by Bardin. Results: The data were categorized into two thematic categories, namely: "How do I perceive pain?" and "Analgesia and pain management". The statements included points such as the perception of pediatric pain, the instruments they use to assess pain and strategies for relief. Conclusion: It was possible to identify that pediatricians and neonatologists are sensitive to the management of pediatric pain, are concerned and prescribe analgesia considering the escalation of medications, the clinical condition and the particularities of each individual. However, it was possible to identify that the participants do not use pediatric pain assessment instruments.
\end{abstract}

Key words: Pain, Analgesia, Pediatrics.

\section{RESUMEN}

Objetivo: Identificar la percepción de los médicos sobre el manejo del dolor y los criterios de elección de analgésicos en pacientes pediátricos y neonatales hospitalizados en unidades de cuidados intensivos pediátricos y neonatales. Métodos: Estudio descriptivo cualitativo realizado en 2020 en una Unidad de Cuidados Intensivos Pediátricos (UCIP) y una Unidad de Cuidados Intensivos Neonatales (UTINEO) de un Hospital Regional de nivel terciario vinculado a una universidad privada del estado de São Paulo. Paulo. Para la realización de las entrevistas se utilizó una entrevista semiestructurada y se analizó de acuerdo al Análisis de Contenido propuesto por Bardin. Resultados: Los datos se clasificaron en dos categorías temáticas, a saber: "¿Cómo percibo el dolor?" y "Analgesia y manejo del dolor". Las declaraciones incluyeron puntos como la percepción del dolor pediátrico, los instrumentos que utilizan para evaluar el dolor y las estrategias de alivio. Conclusión: Se pudo identificar que los pediatras y neonatólogos son sensibles al manejo del dolor

\footnotetext{
${ }^{1}$ Universidade do Oeste Paulista (UNOESTE), Presidente Prudente - SP. *E-mail:drarenaalves@gmail.com
} 
pediátrico, se preocupan y prescriben analgesia considerando la escalada de medicamentos, la condición clínica y las particularidades de cada individuo. Sin embargo, fue posible identificar que los participantes no utilizan instrumentos de evaluación del dolor pediátrico.

Palabras clave: Dolor, Analgesia, Pediatría.

\section{INTRODUÇÃO}

A dor é considerada o quinto sinal vital e seu manejo inclui a mensuração, registro de suas características e implementação de medidas para sua melhora e/ou resolução. Todavia, devido ao seu caráter subjetivo, a avaliação da dor ainda é um desafio para a equipe de saúde na prática assistencial (AMARAL-BASTOS M e SOUZA CF, 2014; SANTOS KH e ESCOBAR EMA, 2015).

$\mathrm{Na}$ criança, situações de dor ainda estão repletas de tabus e mitos, porém, provou-se que o paciente pediátrico apresenta memória para dor, uma vez que a dor não tratada ou tratada inadequadamente pode impactar negativamente em seu desenvolvimento fisiológico e psicossocial (CANDIDO LK e TACLA MTGM, 2015; BLASI DGE, et al., 2015).

Na criança hospitalizada, a avaliação da dor caracteriza-se como um processo complexo, uma vez que o paciente pediátrico pode não conseguir descrever suas percepções com clareza ou, até mesmo, não expressar seu desconforto (ULISSES LO, et al., 2017).

Diante disso, o profissional de saúde tem à sua disposição ferramentas que auxiliam na identificação da dor e possibilitam maior articulação com o paciente e família, facilitando a percepção precoce de desconfortos. Para tanto, a escolha adequada de uma escala ou instrumento em pediatria deve considerar as condições clínicas do paciente, idade, aspectos cognitivos e bases socioculturais (ULISSES LO, et al., 2017; BLASI DGE, et al., 2015).

Porém, embora o uso de escalas seja importante ferramenta para o manejo da dor, o padrão ouro para sua avaliação em crianças deve ser o auto relato sempre que possível, mantendo a identificação de medidas comportamentais apenas para os casos nos quais o paciente não tem capacidade de verbalizar o que sente. Além disso, associar o uso de instrumentos com a avaliação clínica (anamnese e exame físico) e comportamental do paciente pediátrico fornece informações mais fidedignas acerca das características da dor, resultando em intervenções mais eficientes (ULISSES LO, et al., 2017).

Após identificar as características da dor, o próximo passo é planejar e implementar medidas para seu tratamento. No tratamento farmacológico de dor em pediatria, é indicada como primeira opção a via oral. Caso não haja controle da dor por essa via, a segunda escolha seria a endovenosa e, por último, a via intramuscular. A associação de anti-inflamatórios não esteróides (AINES) e paracetamol são as medicações mais indicadas para o início do tratamento em casos de dor leve e moderada. Em situações de dor intensa, associa-se um opióide (RIOS J e ABADESSO C, 2014; WORLD HEALTH ORGANIZATION 2012).

Estratégias não farmacológicas para o tratamento da dor também devem ser utilizadas, como ferramentas lúdicas, apoio emocional e medidas de conforto. Após a implementação das intervenções é extremamente necessário que haja uma avaliação de seus resultados, para que o plano de cuidados possa ser reestruturado e adaptado conforme a resposta de cada paciente (CAMPELO LMN, et al., 2018). Os profissionais que prestam cuidados a esses pacientes necessitam desenvolver constantemente competências e habilidades para identificar detalhes importantes durante a avaliação clínica que possam se relacionar à dor, especialmente em unidades de terapia intensiva, já que muitas vezes as crianças estão menos estáveis (REIS G, 2009).

Embora a dor seja um aspecto fundamental a ser investigado nas avaliações médicas diárias de pacientes pediátricos hospitalizados, tal sintoma ainda é subestimado (CANDIDO LK e TACLA MTGM, 2015). Além da dificuldade na sua mensuração, os profissionais ainda têm problemas na identificação de sinais e sintomas relacionados à dor, utilizando com menor frequência os instrumentos de avaliação (AMARAL-BASTOS M e SOUZA CF, 2014; CAMPELO LMN, et al., 2018). 
Nesse sentido, o presente estudo buscou identificar a percepção da equipe médica acerca do manejo da dor e os critérios para a escolha analgésica em pacientes pediátricos e neonatais hospitalizados.

\section{MÉTODOS}

Estudo descritivo de abordagem qualitativa, realizado no ano de 2020 em uma Unidade de Terapia Intensiva Pediátrica (UTIP) e uma Unidade de Terapia Intensiva Neonatal (UTINEO) de um Hospital Regional de nível terciário do interior do estado de São Paulo.

A população de estudo foi composta por médicos e residentes médicos do programa de Pediatria e Neonatologia no hospital de referência. A população foi selecionada em sua totalidade, exceto, os que estiveram em período de férias ou licença no prazo pré-estabelecido à coleta de dados, resultando em 19 entrevistados.

Para a realização das entrevistas, foi utilizada uma entrevista semiestruturada elaborada pela pesquisadora principal, visando uma melhor identificação da percepção da equipe frente ao fenômeno doloroso.

A entrevista semiestruturada contemplou as seguintes questões: O que é dor para você? Quais sinais na criança você considera como dor? Você utiliza alguma escala de dor, se sim, qual? Quais critérios você utiliza para prescrever analgesia para criança hospitalizada? Qual a sua compreensão sobre manejo da dor?. Como questão acessória foi questionado como a dor poderia interferir no tempo de internação e evolução clínica.

Em caso de aceite os entrevistados assinaram o Termo de Consentimento Livre e Esclarecido. Após o aceite, as entrevistas foram gravadas, transcritas e posteriormente excluídas. Os nomes foram substituídos por siglas aleatórias e não será divulgada nenhuma informação de caráter pessoal.

Para a análise dos dados foi utilizada a Análise de Conteúdo Temática proposta por Bardin seguindo as etapas propostas: a pré-análise; exploração do material e por fim, o tratamento e a interpretação dos dados (MINAYO MCS e DESLANDES SFR, 2012).

O presente estudo foi submetido seguindo a resolução $466 / 12$ que dispõe sobre as normas regulamentadoras das pesquisas que envolvem seres humanos. Não infringiu as normas legais e éticas e teve aprovação Comitê de Ética em Pesquisa (CEP) com CAAE no 35147020.0.0000.5515.

\section{RESULTADOS E DISCUSSÃO}

Em relação à caracterização dos participantes, cinco eram do sexo masculino e 14 do sexo feminino, sendo a média ponderada da faixa etária de 38 anos. Sete participantes possuem formação em pediatria intensivista, sete em pediatria neonatal e cinco residentes em pediatria e neonatologia. $O$ tempo de atuação na área infantil variou entre quatro meses a 42 anos, sendo a média de 10 anos.

A partir da análise do material obtido perpassando as etapas de maneira criteriosa, emergiram duas categorias principais. Para garantir o sigilo e anonimato dos entrevistados foi atribuída a letra "C" seguida de um número para nos referirmos aos entrevistados.

\section{Categoria 1: Como percebo a dor?}

Quando questionados sobre a percepção da dor, os entrevistados, em sua maioria, relacionaram a dor aos estímulos que podem ocasionar um desconforto ao paciente. Definiram a dor como uma sensação desagradável, um incômodo intenso que pode ser traduzido em irritabilidade ao indivíduo, o que vem de encontro com a definição de dor da Associação Internacional para o Estudo da Dor (IASP), que a define como "uma experiência sensorial e emocional desagradável associada à lesão tecidual real ou potencial, ou descrita em termos de tal lesão" (IASP, 1994).

Fizeram associação com a fisiologia da dor, relacionando o fenômeno doloroso à sensação nociceptiva, caracterizando a dor como multidimensional, o que baseado na sua fisiologia, pode ser considerado como um 
sinal de alarme do organismo e está sempre atrelado a fatores sensoriais, afetivos, comportamentais, cognitivos e socioculturais, além da patologia de base (HOCKENBERRY MJ, 2011).

"Eu acho que é um desconforto, um sinal, um sintoma clínico de que tem alguma coisa errada né?" [C1].

"Dor é uma manifestação de desconforto, é uma manifestação de desconforto e se traduz em irritabilidade [pausa]. É uma sensação de desconforto" [C8].

"Dor pra mim, é uma sensação nociceptiva causada, que pode ser algo de alerta do corpo, alguma doença ou alguma sensação que deixa a gente mais alerta a algum problema que está acontecendo" [C16].

Disseram ainda que, a dor não é necessariamente física e que possui componentes psicológicos. Relacionaram a dor ao sofrimento do indivíduo independente de possuir alguma alteração biológica.

Essa relação corrobora com a dimensão psicológica e subjetiva da dor, em que fatores intrínsecos ao paciente e experiências prévias contribuem com a presença e intensidade da dor relatada (DOCA FN et al, 2017).

"Qualquer sofrimento que o ser humano passar pode ser físico, emocional, psicológico. Qualquer tipo de sofrimento mesmo." [C2]

"É uma resposta sensorial a algum estímulo. Não necessariamente uma resposta sensitiva só a estímulo tátil, mas pode ser também relacionado a dor de estímulos psicossomáticos, emocionais" [C15].

A dor da criança faz parte do cotidiano dos profissionais entrevistados e mesmo com os avanços nas evidências científicas, ainda são consideradas como o grupo mais vulnerável e exposto às experiências dolorosas durante a hospitalização (FACCIOLI SC, et al., 2020).

Quando questionados sobre os sinais de dor que identificam, citaram, em sua maioria, as alterações de sinais vitais (SSVV) e expressões faciais que a criança apresenta no momento da avaliação. Essas alterações dos sinais vitais durante o fenômeno doloroso vêm de encontro com a incorporação da dor como quinto sinal vital, tendo em vista que a criança com dor apresenta uma desestabilização dos demais SSVV e a verificação da dor como quinto sinal vital possibilita a identificação de sinais de desconforto e instabilidade (ARAUJO LC e ROMERO B, 2015). Além disso, citaram o autorrelato como meio de avaliação da dor das crianças maiores, como abordagem majoritária de avaliação da dor, no entanto, essa forma de avaliação ainda depende extensivamente da linguagem baseada na palavra falada, e os entrevistados afirmaram terem dificuldades em avaliar a dor da criança quando ainda não consegue se comunicar verbalmente (BREAU LM, 2009).

"É... aumento de frequência cardíaca, queda de saturação, aumento da frequência respiratória, né? Então, alteração dos sinais vitais. Face de dor, por exemplo no recém-nascido, franze a testa. E criança maior, o choro mesmo né? No menor também, mas na criança maior é importante o choro. E na criança maior, o relato né? Ela vai falar mesmo está com dor" [C7].

"Na criança, eu acho que é mais difícil a gente dizer. Normalmente, o choro. Mas as vezes tem uma criança que não consegue ainda se comunicar, que não fala, ela vai se comunicar pelo choro e não necessariamente o choro quer dizer que ela esteja com dor, né?" [C14].

Avaliar a dor infantil exige uma ampla visão e embasamento na multidimensionalidade uma vez que a subjetividade pode prevalecer no momento da avaliação, instituir-se uma escala de mensuração torna-se de suma importância no que tange a assistência qualificada ao usuário (STEVENS B, et al., 2007).

Dessa forma, foi questionado sobre a utilização de instrumentos específicos para a avaliação da dor pediátrica. A maioria dos entrevistados disseram não usar instrumento para avaliação pois no cenário que atuam, não tem nenhuma padronização. 
"Não utilizo. É observacional. Nenhuma escala específica não" [C11].

"Aqui na UTI a gente não usa uma escala propriamente dita" [C16].

"Só os sinais. Não uso escala aqui" [C19].

Alguns entrevistados que afirmaram utilizar instrumentos de avaliação da dor pediátrica não recordaram o nome do instrumento, e entre aqueles que lembraram, os instrumentos citados foram, a escala visual de dor, a FLACC, a COMFORT, RASS ou RANSEI. Entre as falas, foi citado por mais de uma vez a escala das "carinhas".

Dentre as escalas citadas, a Comfort- Behavior (Comfort- B) é descrita como uma alternativa já validada para pacientes pediátricos criticamente doentes, inconscientes e mecanicamente ventilados. Ela contém parâmetros comportamentais e dispõe de um item referente ao choro utilizado especificamente para crianças fora de ventilação mecânica (GRISPAN L, et al., 2014).

Já a FLACC (um acrônimo para: Face, Legs, Activity, Cry, Consolability) é uma escala comportamental utilizada em crianças de 2 meses a 7 anos de idade, considerada um método simples para a identificação e avaliação do fenômeno doloroso (SILVA FC e THULER LCS, 2008).

A escala das "carinhas" citada por alguns entrevistados define-se como a Escala de Faces proposta por Claro (1994) que consiste em uma escala de avaliação adaptada para a população pediátrica composta pelos personagens Cebolinha e Mônica e varia em uma pontuação de zero a quatro, sendo zero sem dor e quatro, a dor mais forte que a criança já sentiu.

"Eu utilizo aquela escala das caretinhas né, do rostinho, que eu esqueci agora o nome. E eu utilizo também dentro da unidade de terapia intensiva, nos pacientes sob sedação, tudo, eu utilizo a COMFORT ou RASS ou RANSEl" [C15].

"Eu utilizo a da carinha, mas eu não lembro o nome dela" [C18].

"Sim. Esqueci o nome agora, mas utilizo sim. Escala de CONFORT, não é? Escala de CONFORT nós usamos" [C10].

"Utilizo a escala de FLACC" [C13].

\section{Categoria 2: A analgesia e o manejo da dor}

Na prática profissional dos pediatras e neonatologistas, a prescrição de analgesia faz parte da rotina diária e foi possível identificar que eles selecionam os analgésicos baseados em suas experiências profissionais, protocolos, particularidades de cada paciente.

O monitoramento das prescrições pediátricas é muito importante diante da quantidade de medicamentos prescritos, sendo que as complicações relacionadas a sua utilização, são o tipo de evento adverso mais comum na internação, representando 3 a $5 \%$ das reações adversas medicamentosas que podem ser prevenidas em hospitais (QUEIROZ KCB, 2015).

Quando questionados sobre os critérios na escolha da analgesia, alguns relatos contemplaram os mesmos sinais que haviam citado na identificação da dor. Outros, complementaram os critérios com diagnósticos, procedimentos dolorosos, situação clínica da criança e presença de dispositivos invasivos.

"Por exemplo, se você... Por exemplo, tem uma criança que tem uma fratura, alguma coisa visível, eu acho que já da para entrar com alguma medicação um pouco mais forte, não por exemplo, só com uma dipirona" [C14].

"Choro, expressão, uma dor abdominal intensa, quando a criança... Quando eu vejo que realmente incapacita de brincar, de ter outras atitudes... Essa criança tem dor! Né? Acho que é isso, só de olhar mesmo" [C2].

"É ... a agitação né, dessa criança, ela pode estar mais agitada então precisando de mais, talvez, analgesia. E a própria presença de dispositivos ou procedimentos 
dolorosos, tipo intubação, dreno, ou procedimento como uma coleta de líquor, gaso etc." [C7].

"Critério? É... pós operatório, né? Para todo e qualquer pós operatório. É... realização de procedimentos invasivos. Ventilação mecânica invasiva, precisa de analgesia. É analgesia né? É... ventilação invasiva, procedimentos dolorosos, pós operatório" [C19].

Alguns participantes relataram realizar um escalonamento das medicações, iniciando pelos analgésicos menos potentes ao mais potente, de acordo com a evolução do quadro clínico da criança e faixa etária. Dentre as falas, foi citado como analgésico menos potente, o paracetamol e dipirona, como analgésico moderado, os anti-inflamatório não esteroidal (AINE) e como analgésico potente, o tramadol, nausedron e morfina. Já para os neonatos, foi citado a glicose a $25 \%$. Esse escalonamento se baseia na Escada Analgésica proposta pela Organização Mundial de Saúde (OMS) que consiste em dois degraus na escolha dos medicamentos de acordo com a intensidade da dor da criança, sendo que, para crianças maiores de três meses, com dor leve, a primeira escolha deve ser entre o paracetamol e ibuprofeno e nas crianças menores de três meses de idade, a recomendação é o paracetamol como escolha única. Já para dores moderadas a severas, a escolha passa a ser a morfina e o fentanil (WORLD HEALTH ORGANIZATION, 2012).

"Critérios de exame físico, choro... é o que eu falei, aumento da frequência cardíaca, você vê que a criança está agitada, é... irritação, movimentação na incubadora, é.... fácies. E aí, a gente primeiro associa uma analgesia leve, que é usual, com dipirona, paracetamol e depois a gente tende a associar com outras analgesias mais fortes" [C4].

"Inicialmente você é ... tudo depende é claro da patologia, né? Mas em média você começa com analgésico simples, as vezes associa um antiinflamatório, quando é necessário, e aí você começa a evoluir com outras ... outros receptores, vamos dizer assim, de dor" [C15].

"Qual critério? ... Se a dor for considerada leve eu uso um analgésico... um analgésico simples, dipirona, paracetamol. Uma dor moderada já um AINE. E uma dor forte opióide, tramadol, nausedron, até morfina" [C6].

"Dependendo do procedimento, se for procedimento doloroso, na UTI neonatal, a gente usa glicose né... a 25\% antes dos procedimentos" [C2].

Também relataram a percepção do manejo da dor, tendo em vista que contempla desde o reconhecimento da dor até o momento em que o profissional define quais estratégias deve utilizar para aliviá-la. Para que essa sequência de eventos possa ser realizada de maneira adequada, o profissional necessita compreender a importância do seu papel nesse processo e reconhecer o quanto está preparado para realizar esse manejo (FACCIOLI SC, et al., 2020). A maioria das entrevistas contemplaram a dificuldade na realização do manejo da dor e o quanto ainda precisa ser estudado, melhorado e colocado em prática nos cenários de hospitalização pediátricos e neonatais.

"Acho que ainda tem muito a melhorar. É.. as vezes, principalmente na NEO, ela é bem subjetiva, principalmente quando o bebê é prematuro, entendeu? É... acho que só assim" [C17].

"Hoje ainda é muito pouco assim... visto nas UTIs isso, né? Então a gente repara que, é ... ou a criança está muito chumbada ou a criança está com pouca analgesia. Eu acho que tem que otimizar isso" [C16].

"Que ela é extremamente necessária, porque é algo que causa desconforto pro paciente, então tem que ser tratado. A gente tem que causar um alívio no paciente né... Ja é um momento ruim estar internado, então qualquer desconforto tem que ser solucionado" [C9]. 
Os entrevistados abordaram a importância da diversidade nas terapias de alívio da dor pediátrica, citando os métodos não farmacológicos como alternativa de escolha, como por exemplo, a acupuntura.

Estudo realizado em unidade de neonatologia identificou a avaliação da dor pela expressão facial e pelo choro do neonato e o tratamento com analgésicos anti-inflamatórios não esteroidais previamente prescritos. Empregaram também métodos não farmacológicos, como mudança de decúbito, massagem local e sucção não nutritiva (WITTI N, et al., 2016).

"Dentro da unidade de terapia intensiva a gente acaba tendo que estudar bastante sobre isso, pra maior conforto do paciente, né? Então a gente acaba tendo bastante respostas com relação a isso. Porém, a gente sabe que tem outras formas da gente minimizar a dor sem ser com fármacos. Cada vez mais está sendo utilizado isso, que na verdade seria a gente fazer aqueles pontos dolorosos, você acabar utilizando eles como, vamos supor, um paciente oncológico, não necessariamente você precisa dar um fármaco, às vezes você pode fazer acupuntura, e assim tende a melhorar" [C15].

"Eu acho que a gente ainda tem que melhorar muito em relação ao manejo da dor. Não podemos esquecer também que existem medidas não medicamentosas né, para tratar a dor. Mas acho que a gente ainda precisa melhorar nessa parte" [C7].

Um ponto importante abordado durante as entrevistas, foi o quanto a dor pode influenciar no prolongamento da hospitalização da criança e os efeitos que ela pode ocasionar a curto, médio e longo prazo.

As falas contemplaram a associação da dor com a manipulação excessiva, procedimentos invasivos, internações prolongadas e até mesmo possíveis traumas para a criança. Essas associações geram reações desagradáveis, como o medo, ansiedade e resistência aos procedimentos que serão realizados. A hospitalização significa agressão a seu mundo lúdico e mágico e, por isso, requer do profissional que a assiste, a compreensão do mundo infantil (ZISK RY, et al., 2015).

Os participantes relacionaram a experiência dolorosa da criança hospitalizada como desencadeadora do estresse e com isso, maior predisposição ao processo infeccioso.

"Eu acho que ela pode levar a vários outros problemas lá na frente, principalmente crianças que tem internação prolongada. E... eu acho que as vezes traz prejuízos lá na frente, dependendo né, não só uma dor simples 'ah, estou com uma dor agora de cabeça' ... Então eu acho que depende muito. Eu acho que dependendo da criança também, se for muito manipulada na hora, dependendo de quanto ela sofrer de dor no momento de estresse, eu acho que isso tem várias consequências" [C14]

"Pode aumentar por que as vezes a dor é intensa e pode ficar mais estressado, pode ter mais predisposição a ter mais infecções, né? E não conseguir desmame ventilatório, pode interferir também" [C4].

"Eu acho que quando a criança, ela tá mais confortável também, com certeza isso melhora o prognostico, diminui o tempo de internação. Acho que diminui, melhora... como eu posso falar... ela vai se curar mais rápido... e diminui tempo de internação e vai embora para casa mais rápido. Né? Então acho que é isso" [C1].

O estresse pode provocar maior percepção de dor por parte da criança, e vice-versa, e desencadear comportamentos mal-adaptativos. Em um estudo realizado mostrou que as três variáveis, estresse, dor e enfrentamento, correlacionaram-se positivamente de modo significativo. Desse modo, é considerada a ideia de que experiências dolorosas podem comprometer o sistema biopsicossocial da criança, produzindo reações estressantes (SILVEIRA KA, 2018).

\section{CONCLUSÃO}

O presente estudo possibilitou identificar que os pediatras e neonatologistas estão sensibilizados com o manejo da dor pediátrica, se preocupam e prescrevem analgesia considerando o escalonamento das 
medicações, o quadro clínico e as particularidades de cada indivíduo. Porém, foi possível identificar que alguns participantes não utilizam instrumentos de avaliação da dor pediátrica, o que pode influenciar no manejo da dor como um todo, tendo em vista que, a avaliação fidedigna da dor é condição sine qua non para um manejo adequado.

\section{REFERÊNCIAS}

1. AMARAL-BASTOS M, SOUSA CF. Valorizar a dor na criança: uma reflexão voltada para a práxis. Nascer e Crescer, $2014 ; 23(4): 190-4$.

2. ARAUJO LC, ROMERO B. Pain: evaluation of the fifth vital sign. A theoretical reflection. Rev Dor, 2015; 16(4): 291 6.

3. BLASI DGE, et al. Avaliação e manejo da dor na criança: percepção da equipe de enfermagem. Semina Cienc. Biol. Saude, 2015; 36 (1): 301-10.

4. BREAU LM. Assessing pain in children with intellectual disabilities. Pain Res Manag., 2009; 14(2):116-20.

5. CAMPELO LMN, et al. A dor da criança com doença falciforme: abordagem do enfermeiro. Rev. Bras. Enferm., 2018; 71 (3): $1381-7$.

6. CANDIDO LK, TACLA MTGM. Avaliação e caracterização da dor na criança: utilização de indicadores de qualidade. Rev. Enferm. UERJ.,2015; 23(4): 526-32.

7. DOCA FN et al. Pain in pediatric inpatients: prevalence, characteristics, and management. Psychol Neurosci., 2017; 10 (4): 394-403.

8. FACCIOLI SC, et al. The management of pediatric pain and the perception of the nursing team in light of the Social Communication Model of Pain. BrJP, 2020; 3(1): 37-41.

9. IASP. Classification of chronic pain. Descriptions of chronic pain syndromes and definitions of pain terms Seattle: IASP Press; 1994; 2: 209-14.

10. HOCKENBERRY MJ. Wong fundamentos de enfermagem pediátrica. 8. ed. Rio de Janeiro: Mosby, 2011; 1: 162202.

11. MINAYO MCS, DESLANDES SFR. Pesquisa social: teoria, método e criatividade. 30. ed. Petrópolis, RJ: Vozes, 2016.

12. QUEIROZ KCB, et al. Análise de interações medicamentosas identificadas em prescrições da UTI Neonatal da ICUHGU. Journal of Health Sciences, 2015; 16(3).

13. REIS G. Avaliação e controle da dor em cuidados intensivos neonatais: experiência do Hospital Pediátrico de Coimbra. Dor, 2009; 17(2):18-23.

14. RIOS J, ABADESSO C. Tratamento da dor moderada aguda na criança. Sociedade Portuguesa de Pediatria. Lisboa, 2014.

15. SANTOS KH, ESCOBAR EMA. O manejo clínico da dor em pediatria: considerações sob a ótica do cuidado em enfermagem. Rev. Enfermagem Revista, Campinas, 2015; 18(1): 101-20.

16. SILVA FC, THULER LCS. Tradução e adaptação transcultural de duas escalas para avaliação da dor em crianças e adolescentes. J Pediatr, 2008; 84(4): 344-9.

17. SILVEIRA KA, et al. Estresse, dor e enfrentamento em crianças hospitalizadas: análise de relações com o estresse do familiar. Rev. SBPH, 2018; 21(2): 5-21.

18. STEVENS B, et al. Determining the structure of acute pain responses in vulnerable neonates. The Canadian Journal of Nursing Research. Revue Canadienne de Recherche en Sciences Infirmieres, 2007; 39(2): 32-47.

19. ULISSES LO, et al. O manejo da dor em crianças percebido pela equipe de enfermagem. Rev. Enferm. UERJ, 2017; 25: $1-7$.

20. WITTI N et al. A guide to pain assessment and management in the neonate. Curr Emerg Hosp Med Rep., 2016; 4: $1-10$.

21. WORLD HEALTH ORGANIZATION. Persisting pain in children package: WHO guidelines on the pharmacological treatment of persisting pain in children with medical illnesses. 2012.

22. ZISK-RONY RY, et al. Nurses' report of in-hospital pediatric pain assessment: examining challenges and perspectives. Pain Manag Nurs., 2015; 16(2): 112-20. 